\title{
An Expert Panel Statement on the Beneficial Effects of Human Milk Oligosaccharides (HMOs) in Early Life and Potential Utility of HMO-Supplemented Infant Formula in Cow's Milk Protein Allergy
}

\author{
Bulent Enis Sekerel ${ }^{1}$ \\ Gulbin Bingol $\mathbb{D}^{2}$ \\ Fugen Cullu Cokugras ${ }^{3}$ \\ Haluk Cokugras ${ }^{4}$ \\ Aydan Kansu ${ }^{5}$ \\ Hasan Ozen ${ }^{6}$ \\ Zeynep Tamay ${ }^{7}$ \\ 'Division of Pediatric Allergy, \\ Department of Pediatrics, Hacettepe \\ University Faculty of Medicine, Ankara, \\ Turkey; ${ }^{2}$ Division of Pediatric Allergy and \\ Immunology, Department of Pediatrics, \\ Acibadem Mehmet Ali Aydinlar \\ University School of Medicine, Istanbul, \\ Turkey; ${ }^{3}$ Division of Pediatric \\ Gastroenterology, Department of \\ Pediatrics, Istanbul University Cerrahpasa \\ Faculty of Medicine, Istanbul, Turkey; \\ ${ }^{4}$ Division of Pediatric Allergy and \\ Immunology, Department of Pediatrics, \\ Istanbul University Cerrahpasa Faculty of \\ Medicine, Istanbul, Turkey; ${ }^{5}$ Division of \\ Pediatric Gastroenterology, Department \\ of Pediatrics, Ankara University School of \\ Medicine, Ankara, Turkey; ${ }^{6}$ Division of \\ Pediatric Gastroenterology, Department \\ of Pediatrics, Hacettepe University \\ Faculty of Medicine, Ankara, Turkey; \\ ${ }^{7}$ Division of Pediatric Allergy and \\ Immunology, Department of Pediatrics, \\ Istanbul University Istanbul Faculty of \\ Medicine, Istanbul, Turkey
}

Correspondence: Bulent Enis Sekerel Division of Pediatric Allergy, Department of Pediatrics, Hacettepe University

Faculty of Medicine, 06100, Sihhiye,

Ankara, Turkey

Tel + 90 312305 1700

$\mathrm{Fax}+903123055000$

Email b_sekerel@yahoo.com

\begin{abstract}
This review by pediatric gastroenterology and allergy-immunology experts aimed to address the biological roles of human milk oligosaccharides (HMOs) and the potential utility of HMOs in prevention of allergy with particular emphasis on cow's milk protein allergy (CMPA). The participating experts consider HMOs amongst the most critical bioactive components of human milk, which act as antimicrobials and antivirals by preventing pathogen adhesion to epithelial cells, as intestinal epithelial cell modulators by enhancing maturation of intestinal mucosa and intestinal epithelial barrier function, as prebiotics by promoting healthy microbiota composition and as immunomodulators by modulating immune cells indirectly and directly. Accordingly, the participating experts consider the proposed link between HMOs and prevention of allergy to be primarily based on the impact of HMO on gut microbiota, intestinal mucosal barrier, immunomodulation and immune maturation. Along with the lower risk of respiratory and gastrointestinal infections, HMOsupplemented formulas seem to be promising alternatives in the management of CMPA. Nonetheless, the effects of individual as well as complex mixtures of HMO in terms of clear clinical and immunological effects and tolerance development need to be further explored to fully realize the immunomodulatory mechanisms and the potential for HMOs in prevention of allergic diseases and CMPA.
\end{abstract}

Keywords: human milk oligosaccharides, gut microbiota, intestinal mucosal barrier, infant formula, immune tolerance, cow's milk protein allergy

\section{Introduction}

The nutritional composition and the non-nutritive bioactive content of human milk are unique for survival and healthy development of the human infant. ${ }^{1}$ Accordingly, infant feeding is recommended to include exclusive breastfeeding during the first six months of life along with continued breastfeeding for 1 to 2 years of life with complementary feeding. ${ }^{1}$

Although infant formula milk is an alternative for infant nutrition when human milk is not available, the formula-fed versus exclusively breastfed infants are considered to be more vulnerable to infectious and immune diseases. ${ }^{2}$

Human milk oligosaccharides (HMOs) are the third-most abundant solid component in maternal milk following the lipids and lactose. ${ }^{3-5}$ HMOs are associated with certain physiological functions and clinical benefits such as the modulation of 
microbiota composition, modulation of gut epithelial cell responses and immunomodulatory and anti-inflammatory effects. $^{3-5}$ In this regard, HMOs have become the focus of current scientific research, given that they offer further optimization of infant nutrition by reducing the compositional difference between human milk and infant formula, as a key support to immune development in early life. ${ }^{6-10}$

Nonetheless, while the technological advances enabled the addition of up to 5 oligosaccharides structurally identical to HMOs in infant formula, the oligosaccharides composition from infant formula remains very different from human milk, both in diversity and concentration. ${ }^{7,11}$

There is an emerging evidence in humans in favor of the role of infant gut microbiome in food sensitization/allergy.12,13 Given the benefits of HMOs in terms of early intestinal microbiome development and gastrointestinal and systemic immune modulatory functions, ${ }^{5,6,14-16}$ supplementation of infant formula with HMOs has been considered a promising strategy in non-exclusively breastfed infants including those with cow's milk protein allergy (CMPA). ${ }^{9,17}$

Although based on scarce amount of literature data in infants, HMO-supplemented infant formula was reported to be a safe and tolerable supplementation with several positive effects. $^{7,9,17}$ Amongst these are the increased amount of bifidobacteria and short chain fatty acids (SCFAs) production, likelihood of reduction in respiratory tract infections and provision of an accepted hypoallergenicity criteria, when added to existing extensively hydrolyzed (eHF) or amino acid-based (AAF) formula in CMPA. ${ }^{7,9,17}$

Therefore, this review by a panel of pediatric gastroenterology and allergy-immunology experts aimed to address the biological roles of HMOs in terms of early gut microbiota development and gastrointestinal and systemic immunomodulatory properties and the potential utility of HMOs in prevention of allergy with particular emphasis on infants and children with CMPA.

\section{Methods}

The present expert panel consisting of pediatric gastroenterology and pediatric allergy-immunology specialists who are members of the Turkish Society for Pediatric Gastroenterology, Hepatology, and Nutrition and the Turkish National Society of Allergy and Clinical Immunology Society met to develop consensus opinion on the biological roles and potential utility of HMOs in infants and children with CMPA. The participating experts (professors, also international speakers and national influencers), who had at least 15 years of experience in pediatric gastroenterology and pediatric allergyimmunology were invited after being recommended by the presidents of the Turkish Society for Pediatric Gastroenterology, Hepatology, and Nutrition and the Turkish National Society of Allergy and Clinical Immunology Society. The panel performed a broad nonstructured literature search to reveal the potential studies and agreed on the studies to be included in the review. The panel critically analyzed the evidence-based published literature regarding biological roles and efficacy of HMOs in breastfed infants according to HMO composition as well as the clinical trials with use of HMO-supplemented infant formula in prevention of allergy and CMPA in particular. Based on the available scientific evidence and the experts' clinical opinion, this consensus document comprehensively addresses HMOs in terms of a) overview of human milk bioactive components, b) structure and biological roles of HMOs, c) advantages of HMOsupplemented infant formula, and d) rationale and potential benefits of using HMO-supplemented infant formula in prevention of allergy with special emphasis on infants and children with CMPA.

\section{HMOs: one of the most important bioactive components of human milk}

The functional or bioactive components of human milk, the elements affecting the biological processes or substrates and ultimately the health, have increasingly been recognized as factors responsible for the short- and long-term benefits of human milk feeding. ${ }^{18,19}$ These functional components involve a large group of several compounds such as protein (lactoferrin), carbohydrates (especially HMOs), fats (polyunsaturated fatty acids), immunoglobulins, hormones, vitamins, nucleotides, minerals, prebiotics, probiotics, transporters, antioxidants and antimicrobial factors. ${ }^{18}$

T-helper (Th) cell population is still biased towards the Th2 phenotype (humoral immunity) rather than Th1/Th17 phenotype (cellular immunity) at birth. ${ }^{20-22}$ Human milk provides protection during this vulnerable period via bioactive components that stimulate healthy intestinal microbial diversity (Bifidobacteria and Lactobacillus species), prevent pathogenic infection, promote intestinal development and mucosal barrier function, facilitate immune tolerance and directly support the further development of the immune system (Figure 1). ${ }^{5,8,10,15,23-25}$

The physical barriers and the crosstalk between mucosal epithelial cells and underlying immune cells are 


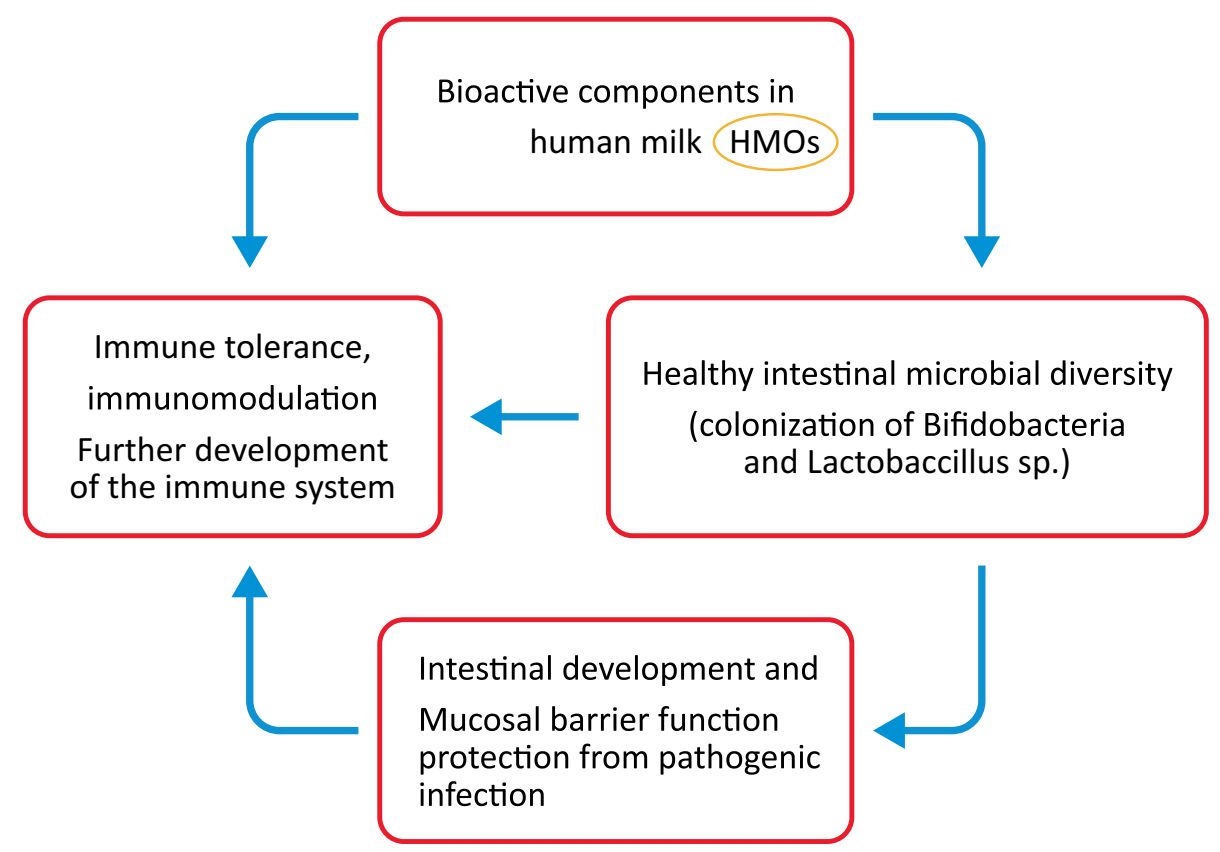

Figure I Human milk bioactive components in relation to provision of healthy neonatal microbial colonization, fine-tuning of inflammatory processes and immune defense and maturation in early life.

considered to play a crucial role in the first-line defense in early life. ${ }^{8,22,26,27}$ Hence, the gastrointestinal tract, as a functional barrier preventing pathogenic invasion and an immune-modulator providing active elements of the lymphoid cell line, is considered to be of utmost importance to the neonatal immunity. ${ }^{21,22}$

The breastfeeding as a nutrient source in early life and particularly the non-digestible HMOs amongst the diverse bioactive components in human milk are considered critical for healthy neonatal microbial colonization, fine-tuning of inflammatory processes and immune defense and maturation in the first years of life (Figure 1). ${ }^{8,10,22}$

The incidence as well as severity of several infectious diseases (ie, bacteremia, diarrhea, otitis media, respiratory and urinary tract infections, necrotizing enterocolitis) and immune system disorders (ie, inflammatory bowel disease [IBD], allergy, asthma, type 1 diabetes) is considered to be lower in breastfed infants vs formula-fed infants. ${ }^{2,7,10,18,28-30}$

Human milk, during the time of complementary food introduction, is considered likely to prevent celiac disease or allergy through interactions between dietary antigens, human milk components and gut associated lymphoid tissue (GALT). ${ }^{31-34}$

\section{Structure and biological roles of HMOs}

Human milk is a complex matrix and a living tissue consisting of $87 \%$ water, $3.8 \%$ lipid, $1.0 \%$ protein and $7 \%$ lactose, while HMOs (1-1.5\%) represent the third largest component of human milk, after lactose and lipids. 5,7,11,35,36

HMOs are a unique mixture of structurally diverse indigestible carbohydrates present in the human milk..$^{7,10,19,37}$ The concentration and composition of HMOs is subjected to inter-individual variation, depending on the genetic predisposition and geographical origin of mothers as well as on the gestational age at delivery (higher concentrations but lower levels of fucosylated HMOs in preterm vs term milk), stage of lactation (colostrum: 20-25 g/L; mature milk: 5-20 g/L) and maternal nutrition. ${ }^{7,8,10,11,22,38,39}$

The changes in HMOs from secretor mothers during the course of lactation was reported to include a decrease in total, fucosylated, sialylated, non-fucosylated neutral $\mathrm{HMO}$ concentrations over time, an increase in the percentage of fucosylation and a decrease in the percentage of sialylation, while non-fucosylated neutrals are considered to remain stable over time. ${ }^{40}$ Hence, HMOs do not decrease in concentration uniformly across lactation, with change in individual HMO concentrations during lactation and predominance of different HMOs in each stage. ${ }^{40,41}$

2 '-fucosyllactose $\left(2^{\prime}\right.$-FL) is the most abundant HMO, accounting for about $20-40 \%$ of total HMO concentration in colostrum, whereas $6^{\prime}$-sialyllactose $\left(6^{\prime}-\mathrm{SL}\right)$ is the dominant form of sialylated HMO at the early stages of lactation, followed by $3^{\prime}$-sialyllactose ( $\left.3^{\prime}-\mathrm{SL}\right)$ in late lactation. ${ }^{40}$ HMOs are considered to be stable over one-week during 
early lactation, while most HMOs decline as lactation progresses, except for 3'-FL and 3'-SL which are consistently higher at 6 months and 12 months of lactation. ${ }^{40-43}$

Indeed, the efforts to enhance the quality of infant formulas aim not only to mimic the composition of human milk in every respect (ie, adapting the composition of bioactive compounds besides the concentration of macronutrients and micronutrients) to make it as similar as possible to human milk but also to achieve physiological effects as in breastfed infants. ${ }^{18}$ Hence, whether the HMO supplementation ultimately improves infant formula milk in a way that mimics the documented benefits of human milk remain to be further investigated. ${ }^{42,44}$

\section{Structure of HMOs}

Of at least 200 unique HMOs identified in human milk, more than 100 were structurally defined. ${ }^{38,45,46}$ HMOs consist of monosaccharides and monosaccharide derivatives with a lactose, polylactosamine or lacto-N-biose core, while a further modification through differently linked fucose or sialic acids to the molecule enables structural diversity and resistance against enzymatic digestion. ${ }^{22}$

The basic HMO structure is fucosylated or nonfucosylated and/or sialylated, resulting in neutral, neutral N-containing and acidic HMO subtypes (Figure 2). 5,7,47,48

The neutral HMOs comprise $>75 \%$ of the total HMOs in the human milk, while the most abundant HMO is the $2^{\prime}-\mathrm{FL}$
(2.7 g/L, range, 1.88-4.9 g/L). ${ }^{7,39} 2^{\prime}$-FL, a glucose, galactose, and fucose based trisaccharide, constitutes almost $30 \%$ of the total HMOs in secretor mothers (Figure 2). ${ }^{7,39}$

HMO profile of human milk is dependent largely on the individual maternal genetic disposition. ${ }^{8}$ Hence, the complex HMO composition is genetically determined by the activity of the secretor (Se) fucosyltransferase 2 gene encoding $\alpha 1$-2-fucosyltransferase (FUT2) and the Lewis (Le) gene encoding $\alpha 1-3 / 4$-fucosyltransferase (FUT3), and is regulated by glycosyl-transferases within the mammary gland. 5,31,34,49

- Based on Se locus, women are categorized into secretors (those with functional FUT2 enzyme and with 2'-FL, LNFP I and other $\alpha 1$-2-fucosylated HMOs in milk) and non-secretors (those lack a functional FUT2 enzyme and with no $\alpha 1$-2-fucosylated HMOs in milk). 7,47,50,51

- Based on Le locus, women are categorized into Lepositive women (express FUT3, milk contain specific 1-4-fucosylated HMOs such as LNFP II) and Lenegative women (lacks FUT3 activity and specific a1-4-fucosylated HMOs in milk). ${ }^{7,47,50,51}$

- Hence, based on the expression of FUT2 and FUT3, the human milk is classified as: Le-positive secretors (Le $+\mathrm{Se}+$ ), Le-negative secretors (Le-Se+), Le-positive nonsecretors (Le+Se-), and Le-negative nonsecretors (Le-Se-) (Figure 3). ${ }^{\text {7,47,50,51 }}$

\section{Neutral (fucosylated) Neutral N-containing HMOs (nonfucosylated) HMOs}

Acid (sialylated) HMOs

\section{Characteristics}

$\%$ in total $\mathrm{HMO}$

$35-50 \%$

$42-55 \%$

$12-14 \%$

Molecule at

fucose

$\mathrm{N}$-acetylglucosamine

sialic acid

terminal position

Individual examples

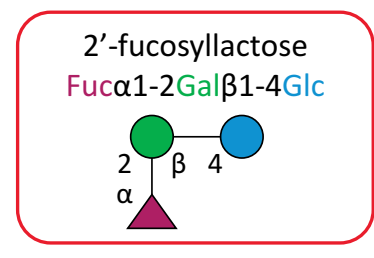

2'-fucosyllactose (2'FL)

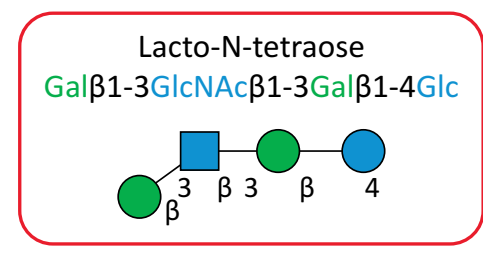

Lacto-N-tetraose (LNT)
6'-Sialylyllactose

NeuAco2-6Galß1-4GIc

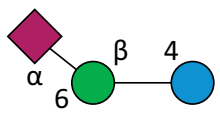

6'-sialyllactose

HMO: Human milk oligosaccharide

Figure 2 Human milk oligosaccharide structural subtypes.

Abbreviation: HMO, human milk oligosaccharide. 


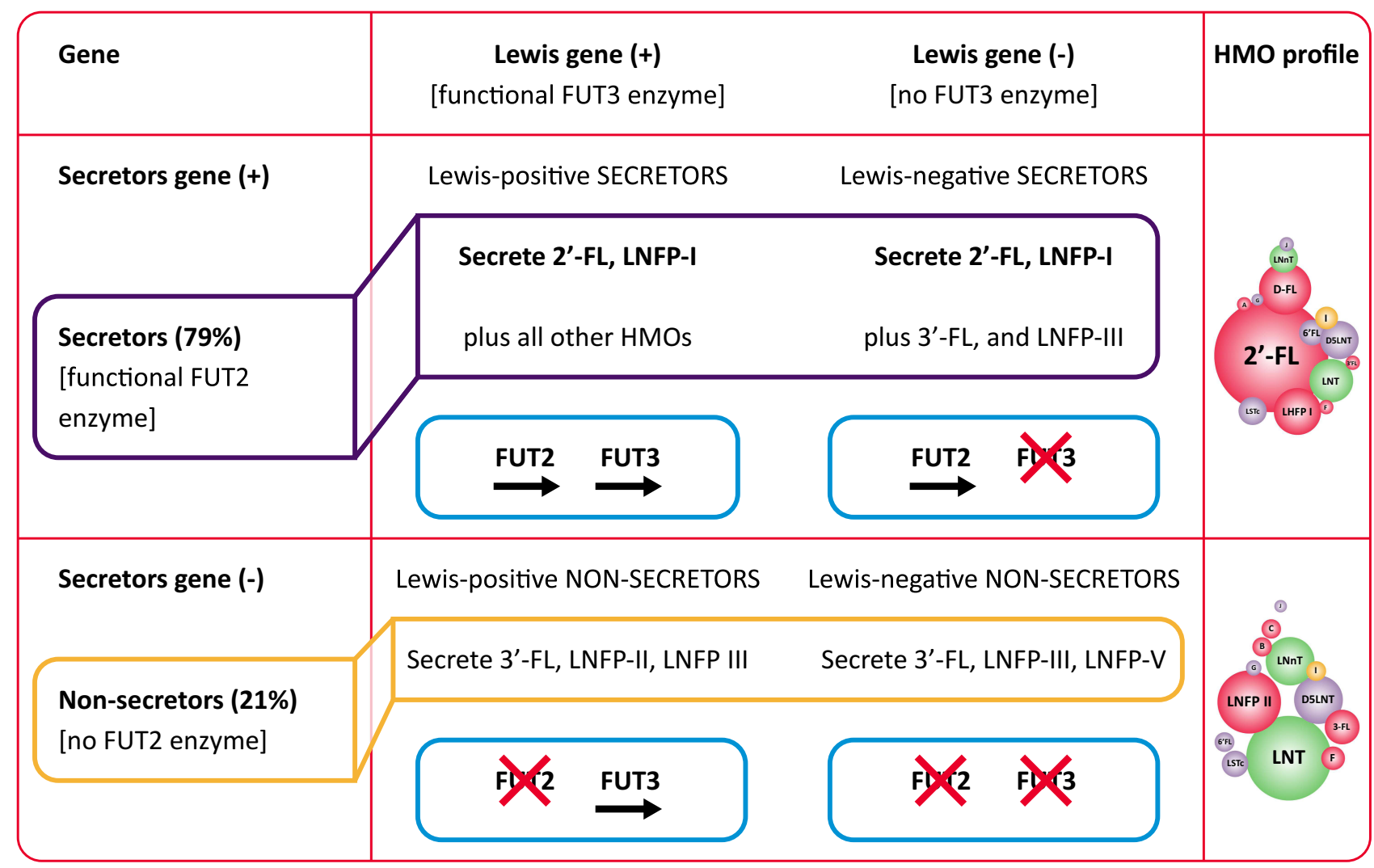

Figure 3 Human milk oligosaccharide profile according to genetic background.

Abbreviations: HMO, human milk oligosaccharide; 2'-FL, 2'-fucosyllactose; LNFP, lacto-N-fucopentaose.

Overall, 79\% of mothers in European and North American populations have the active gene for FUT2 (secretors), while $21 \%$ of mothers lack the functional FUT2 enzyme (non-secretors) and produce milk without $2^{\prime} \mathrm{FL}$ and LNFP I. ${ }^{7,37-39,47}$ Notably, the delayed bifidobacteria colonization and altered microbiota-related metabolic activity and increased risk for diarrheal diseases have been reported in infants fed by non-secretor mothers. ${ }^{10,13,52,53}$ Nonetheless, it should be noted that HMOs differ in terms of individual structures and related effects, emphasizing the importance of balanced complex mixture. ${ }^{31}$

\section{Biological roles of $\mathrm{HMOs}$}

HMOs have several functions, including selectively enriching gut bacteria and thereby promoting healthy microbiota composition, preventing pathogen adhesion to epithelial cells, enhancing maturation of intestinal mucosa and intestinal epithelial barrier function, modulating immune cells, pathogen recognition receptors as well as signaling pathways related to maturation of lymphoid tissue, influencing cytokine and chemokine networks that regulate Th1/Th2 lymphocyte balance and preventing infection and supporting immunity (Figure 4). 6,8,10,15,22
Immunomodulation is one of the most outstanding functions of HMOs, including the indirect modulation of immune responses via changes in infant gut microbiota composition, and the direct effect on immune cells at a systemic level, after reaching the blood circulation (Figure 4). ${ }^{22,46,47,54,55}$

Following gastrointestinal absorption, up to $1 \%$ of HMOS become available in systemic circulation at a level (ranging 10 to $100 \mathrm{mg} / \mathrm{mL}$ ) being sufficient for interaction with cell receptors. ${ }^{46,47,54}$

Therefore, HMOs are considered to have multiple beneficial effects in a breast-fed infant: ${ }^{11}$

- As prebiotics they are metabolic substrates for beneficial bacteria enabling a growth advantage for them over potential pathogens;

- As antiadhesive antimicrobials they act as soluble glycan receptor decoys preventing the pathogenintestinal mucosa contact;

- By directly affecting intestinal epithelial cells and modulate their gene expression, resulting in modifications in the cell surface glycans and other cellular responses; 


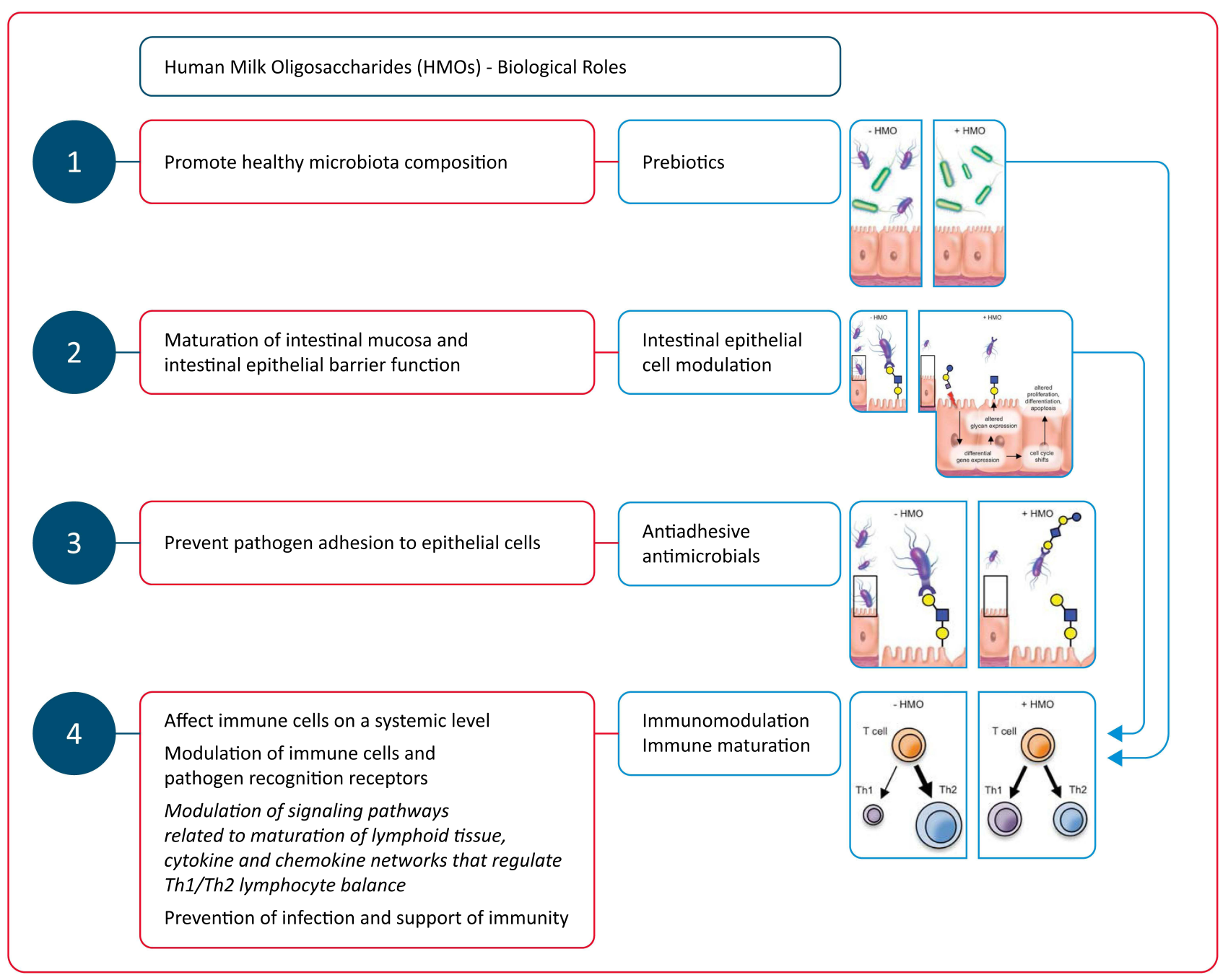

Figure 4 Biological roles of human milk oligosaccharide (HMOs).

Notes: Adapted from Bode L. Human milk oligosaccharides: every baby needs a sugar mama. Glycobiology. 2012;22(9):1।47-II62, by permission of Oxford University Press. ${ }^{\text {I. }}$

Abbreviation: $\mathrm{HMO}$, human milk oligosaccharide.

- By modulating lymphocyte cytokine production, increasing the likelihood of a more balanced Th1/ Th2 response;

- By reducing selectin-mediated interactions between immune cells and decreasing the leukocyte rolling on activated endothelial cells, decreasing the likelihood of mucosal leukocyte infiltration and activation.

HMOs as antimicrobials and antivirals

HMOs mimic the glycans on the gut epithelia that acts as surface pathogen receptors (virus, bacteria, toxins and/or eukaryotes) and they prevent gut epithelial adhesion and biofilm formation through competitive interaction with pathogens. ${ }^{8,31,47,56}$ In addition to acting as soluble decoy receptors and blocking the attachment of viral pathogens to the epithelial cells, HMOs can also indirectly prevent viral adhesion through binding to the epithelial surface causing a structural change in the receptor. ${ }^{57}$ Accordingly, antimicrobial and antiviral effects of HMOs depend on blockade of pathogen access via pathogen or epithelial surface receptor binding to prevent their replication and subsequent infection. ${ }^{8}$ In addition, they are more advantageous than pathogens in terms of being fermented by commensal bacteria (ie, Bifidobacteria) which promote their growth while pathogens experience growth suppression. ${ }^{8,31}$

Notably, in addition to serving as prebiotics, HMOs possess bacteriostatic and antimicrobial properties against 
Streptococcus agalactiae and Gram-negative aerobe Acinetobacter baumannii as well as antibiofilm properties against methicillin-resistant Staphylococcus aureus (MRSA). ${ }^{57,58}$ HMOs were reported to inhibit Campylobacter colonization and to reveal protection against pneumococcal pneumonia in animal studies. ${ }^{59,60}$ The studies in infants revealed that they offer protection against infectious diarrhea, necrotizing enterocolitis, and can directly inhibit the growth of group B streptococcus (GBS), a leading cause of invasive bacterial infection in newborns. ${ }^{61-64}$ Moreover, HMOs are suggested to potentiate the actions of aminoglycosides, anti-folates, macrolides, lincosamides, and tetracyclines against GBS, $S$. aureus and A. baumannii. ${ }^{57,65}$ Hence, prophylactic and therapeutic use of HMOs is also possible in immune compromised infants as well as in those at high risk of infection. $^{10}$

\section{HMOs as intestinal epithelial cell modulators}

HMOs, through interaction with glycans on the epithelial or dendritic cell surface, promote gut maturation and contribute to the barrier integrity via increased growth of Bifidobacterium. ${ }^{8,66}$ HMOs can also directly affect the intestinal cell response by suppressing the cell growth and by stimulating the differentiation and apoptosis, required for a proper intestinal barrier function. ${ }^{7,67}$

In fact, besides their impact on microbes and their roles as decoy receptor for pathogens, HMOs can also directly alter epithelial cell gene expression and thus alter the binding ability of certain pathogens to the cell surfaces via changing the expression of cell surface glycocalyx..$^{3,6,68}$ In addition, HMOs induce production of SCFAs important for gut health such as butyric acid and propionate which can stimulate mucin release, mucosal blood flow and intestinal epithelial cell maturation through their interaction with the host epithelium. ${ }^{8,69-71}$ These functions are considerably important in early neonatal life given that intestinal health and barrier function are the first line of defense in innate immunity. ${ }^{72}$

\section{HMOs as prebiotics and effectors of the microbiota composition}

Since infants lack glycoside hydrolases and intestinal membrane transporters, the majority of HMOs reach the colon undigested and are utilized by certain microbes influencing the gut microbiota. ${ }^{6,73}$ Hence, HMOs are "prebiotics" that selectively induce the growth of beneficial (probiotic) organisms such as Bifidobacterium, a dominant species in the intestine of breast-fed infants. ${ }^{6,19,73}$ Bifidobacteria have the ability to utilize HMOs through specifically expressed glycoside hydrolases and therefore considered to be co-evolved with HMOs. ${ }^{6}$

Accordingly, for breast-fed infants, bifidobacteria can preferentially colonize the infant GI tract by the third month of life. ${ }^{74}$ HMOs also play a role in improved epithelial barrier function by inducing the $B$. infantis growth in the infant gut, which is responsible for production of peptides that normalize intestinal permeability. ${ }^{10,74,75}$ Indeed, a highly selective, prebiotic effect of HMOs has been reported in shaping the gut microbiota in the first weeks of life with a decrease in fecal HMOs via a shift in fecal bacterial population from the nonHMO-consuming microbes (Enterobacteriaceae and Staphylococcaceae) to HMO-consuming bacteria (Bacteroidaceae and Bifidobacteriaceae) ${ }^{74}$

In this regard, HMOs are considered to play a key role in healthy development of gut-microbiota, and thus the intestinal wall barrier and immune functions. ${ }^{6,76}$ Given the impact of maternal secretor status on the infant gut bifidobacterial community, the combination of HMOs with specific bacteria has been suggested likely to modulate gut immunity and gut integrity. ${ }^{31,52}$

HMOs as immunomodulators and potential inducers of immune maturation

The gut barrier involves a mucus layer, epithelium, the resident microbiota, and resident or recruited immune cells, whereas the immune responses are immature in newborns as based primarily on a Th2 type response rather than a Th1 type immune response along with a relative lack of immunological memory., ${ }^{6,77}$

HMOs are considered to affect expression of several cytokines including IL-8, IL-1 $\beta$, colony-stimulating factor 2 (CSF2), platelet factor 4 (PF4) and IL-17C. They also influence the expression of certain chemokines including CXCL1,2,3,6, CX3CL1, CCL5 and CCL20 as well as that of cell surface receptors including intercellular adhesion molecule-1 (ICAM-1), intercellular adhesion molecule-2 (ICAM-2), interferon $\gamma$ receptor 1 (IFNGR1), and IL-10 receptor a (IL10RA). ${ }^{5}$ In this way, HMOs can modulate the intrinsic expression of cell trafficking-related inflammatory markers, the lymphoid tissue-related signaling pathways and the cytokine and chemokine networks responsible for Th1/Th2 lymphocyte balance (Figure 4). ${ }^{8,10,15,78}$

HMOs are considered to target expression of receptors involved in pathogen recognition, such as toll-like 
receptors (TLRs), to interact with dendritic cells (DCs) in close proximity to the intestinal epithelial barrier that leads to $\mathrm{T}$ cell differentiation and/or $\mathrm{T}$ cell/B cell interaction and to show systemic effects by interaction of neutrophils, lymphocytes and monocytes with endothelial cells that affects immune cell populations and secretion of cytokines. $^{8,10,78}$

In fact, DCs are considered to play a cardinal role in the regulation and development of innate and adaptive immune responses during infections and inflammatory diseases and one sub-population, so-called tolerogenic DC (tDC), results in a reduced inflammatory cytokine production (ie, IL-4, IL12, IL-6, and TNF- $\alpha$ ) but an elevated regulatory cytokine production (ie, TGF- $\beta$ IL-10 and IL-27). ${ }^{78}$

Overall, interactions with these chemokines, cytokines, and cell surface receptors are considered to enable subsequent immunomodulation and immune maturation through development of a balanced and efficient immune response and thus a lesser risk of infections and allergic diseases. ${ }^{5,8,10,78}$
Accordingly, HMOs play a role in the maturation of the infant immune system both at the gut barrier level through effects on microbiota, gene expression and intestinal epithelial cell maturation as well as by exerting direct immune modulating effects after being absorbed intact into the systemic circulation. ${ }^{10,22,46,79}$ Notably, nearly $70 \%$ of gastrointestinal immune cells may associate directly with HMOs consumed by infants, while the first few weeks of life represent a key window of immune development with a circulatory peak of innate immune cells during this period. ${ }^{80}$

\section{HMO-supplemented infant formula}

Milk oligosaccharides in human milk present at a higher concentration and a greater structural diversity and degree of fucosylation than those in cow's milk and infant formulae. ${ }^{10}$ HMOs are unique for human milk and not found in the same composition and diversity in animal milk (Figure 5). ${ }^{11,81}$
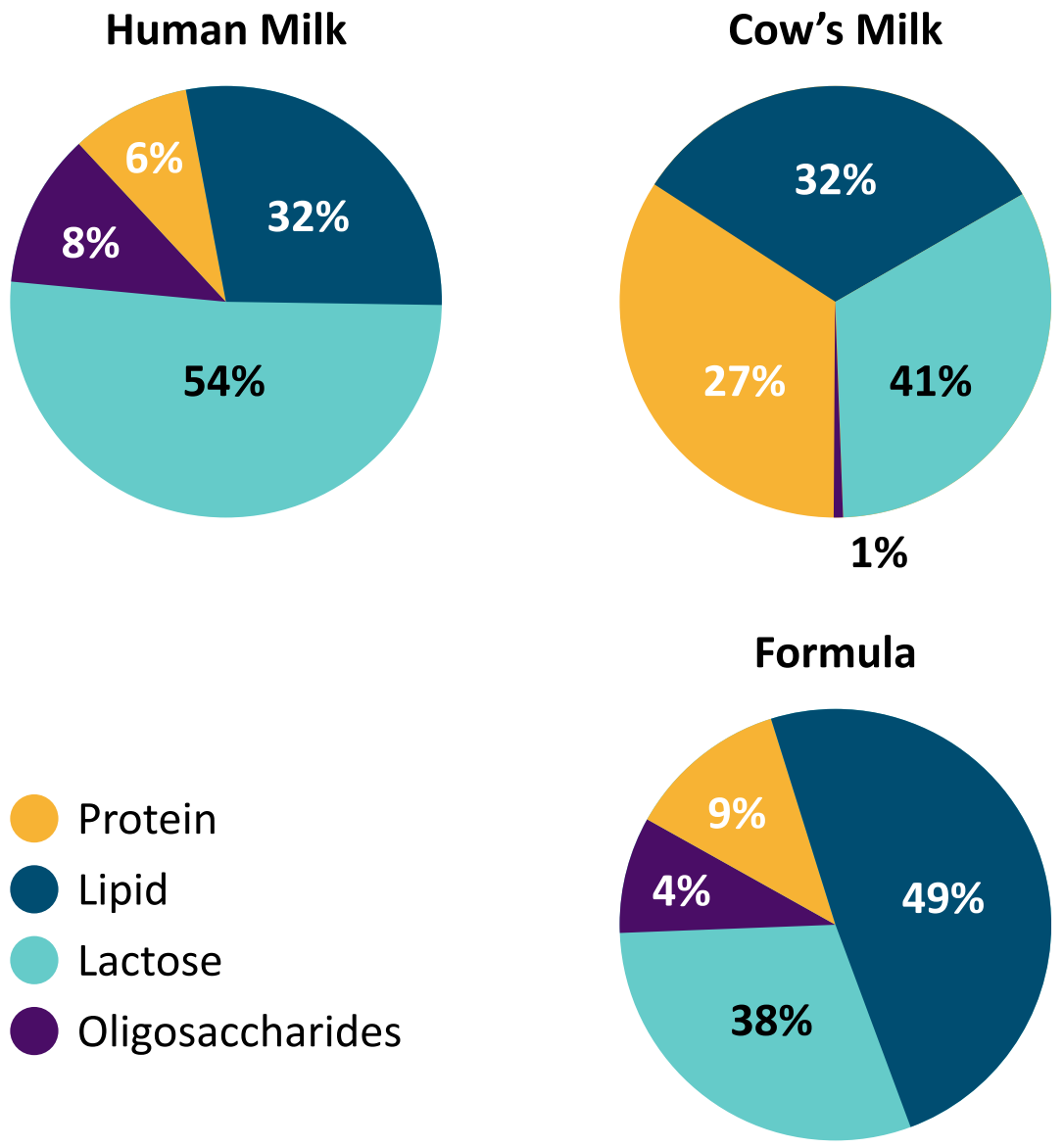

Figure 5 Composition of human milk, formula (Similac ${ }^{\circledR}$ infant formula, Abbott nutrition) and cow's milk. Data from Bode L. ${ }^{\prime \prime}$ 
Despite the well-known benefits of HMOs, it has only recently become technically feasible to produce HMOs identical to human milk HMOs. ${ }^{9}$ Accordingly, cow-milk derived infant formulas have been supplemented with affordable non-digestible carbohydrates (non-HMOs) such as fructo-oligosaccharides (FOS) and galacto-oligosaccharides (GOS) to enable some of the HMO functions. ${ }^{82}$

The recent availability of synthesized 2 '-FL, structurally identical to the $2^{\prime}$-FL in human milk, is important in this regard, enabling the availability of HMOs in some commercial infant formulas. ${ }^{66}$ Currently, two types of HMOs are used as add-on to the infant formula including a fucosylated HMO, 2'FL, and a neutral, non-fucosylated HMO, LNnT. ${ }^{9,47,83}$ HMO-supplemented infant formulae are considered to be safe and beneficial for human infants along with increased availability of commercially produced HMOs. ${ }^{17,79,84}$

$2^{\prime}$-FL is the most abundant HMO in the milk of most lactating women with extensive data on the potential systemic effects. ${ }^{80}$ Preclinical data indicate $2^{\prime}$-FL to have multiple functions via prebiotic, anti-infective, antiinflammatory and immunomodulatory properties and to reduce the risk of necrotizing enterocolitis. ${ }^{80}$ Observational studies revealed the association of 2'-FL HMOs with bifidobacteria dominated early gut microbiota in breastfed infants. ${ }^{52,85,86}$

Albeit the amount of data available on HMO supplementation in infant formula from clinical trials in infants is still limited, ${ }^{4}$ clinical data showed the supplementation of infant formula with $2^{\prime}$-FL to be safe and welltolerated. ${ }^{17,79,84,87,88}$ Moreover, $2^{\prime}$-FL is considered to show similar absorption and excretion characteristics with $2^{\prime}$-FL in human milk ${ }^{84}$ and is associated with similar immune benefits with the breastfed reference group, and fewer respiratory infections. ${ }^{79}$

In a past study with 420 healthy term infants by Marriage et $\mathrm{al}^{84}{ }^{84}$ authors reported no significant differences between groups of infant formulas added with GOS/L (2.4 g), 2'-FL (0.2 g) + GOS/L (2.2 g) or $1 \mathrm{~g} \mathrm{2'-}$ FL $(1 \mathrm{~g})+\mathrm{GOS} / \mathrm{L}(1.4 \mathrm{~g})$ and the breast-fed reference group in terms of data on weight, length, and head circumference. All formulas were well tolerated along with similar 2'-FL relative absorption and excretion between infants fed with $2^{\prime}$-FL containing formulas and the breastfed infants. ${ }^{84}$

Further analysis of 201 healthy term infants from the cohort from Marriage et al, ${ }^{84}$ by Goehring et al, ${ }^{79}$ revealed the circulating cytokines concentrations in infants fed with the 2 '-FL containing formulas to differ from the concentrations in infants fed with GOS containing formulas but not from the concentrations in breast-fed infants. ${ }^{79}$

In a study by Puccio et $\mathrm{al}^{17}$ in healthy infants randomized to a cow's milk-based infant formula (control, $\mathrm{n}=$ 87) or the same formula with $1.0 \mathrm{~g} / \mathrm{L} 2^{\prime}$ fucosyllactose $\left(2^{\prime} \mathrm{FL}\right)$ and $0.5 \mathrm{~g} / \mathrm{L}$ lacto-N-neotetraose (LNnT) (test, $\mathrm{n}=$ 88), the authors reported that weight gain through 4 months, digestive symptoms and behavioral patterns were similar in both groups, except for softer stool and fewer nighttime wake-ups in the test group at 2 months. Infants receiving test (vs control) had significantly fewer parental reports of bronchitis through 4 (2.3\% vs $12.6 \%)$, $6(6.8 \%$ vs $21.8 \%)$, and 12 months (10.2\% vs $27.6 \%)$, lower respiratory tract infection (adverse event cluster) through 12 months $(19.3 \%$ vs $34.5 \%)$, lower rate of antipyretics use through 4 months (15.9\% vs $29.9 \%)$ and antibiotics use through $6(34.1 \%$ vs $49.4 \%)$ and 12 months $(42.0 \%$ vs $60.9 \%) .{ }^{17}$

In a real-world study by Roman et $\mathrm{al}^{87}$ on growth and tolerance in three groups of infants $(n=159)$ including a healthy, exclusively breastfed infants, an exclusively formula-fed group who received a milk-based formula with $2^{\prime}$ FL $(1 \mathrm{~g} / \mathrm{L})$ and $\mathrm{LNnT}(0.5 \mathrm{~g} / \mathrm{L})$, and a group mixed fed with both formula and human milk for 8 weeks, the authors reported that mean z-scores for growth and gastrointestinal tolerance were similar between all groups and incidence of AEs was low overall, and comparable across groups. ${ }^{87}$ Hence, the authors concluded that the growth and tolerance outcomes of a HMO-supplemented infant formula were similar to data from randomized clinical trials, supporting the effectiveness of this early feeding option. ${ }^{87}$

In a prospective, randomized, multi-center, doubleblinded, controlled tolerance study by Kajzer et $\mathrm{al}^{88}$ in 131 healthy term infants assigned to control formula without oligosaccharides $(\mathrm{n}=30)$, formula with $0.2 \mathrm{~g} 2^{6}$-FL/L + $2 \mathrm{~g} \mathrm{scFOS} / \mathrm{L}(\mathrm{n}=35)$ and breast-fed reference group $(n=36)$, the authors noted that 2 -FL and scFOS containing formula was safe and well tolerated with no significant differences among the three groups at 35 days of age in terms of stool consistency, formula intake, anthropometric measures, and percent feedings with spit-up/vomit associated with feeding. ${ }^{88}$

Overall, 2'-FL supplementation of infant formula has been considered to be safe and tolerable approach along with absorption and excretion efficiency similar to 2 -FL in human milk. ${ }^{80}$ The $2^{\prime}$-FL supplementation has also been considered to offer additional advantages such as immune 
benefits, improved tolerance and lower risk of respiratory infections. ${ }^{80}$ Addition of $0.2 \mathrm{~g} 2^{\prime}$-FL/L to the infant formula supports immune and gut health and brings it closer to human milk not only compositionally but also functionally. ${ }^{80}$

\section{Utility of HMO-supplemented infant formula in prevention of allergy Rationale behind the proposed link between HMOs and prevention of allergy} Exclusive breastfeeding has a potential to reduce the development of several diseases such as asthma, allergies, IBD, and type 1 diabetes, ${ }^{15}$ while the "secretor type" related complex mixtures of HMOs are also considered likely to have a critical role in lowering the risk of allergic disease experienced by breast-fed infants later in life. ${ }^{31,89}$
HMOs are suggested to improve gut epithelial integrity, apoptosis, and intestinal permeability and to have indirect and direct immunomodulatory properties. ${ }^{6,15}$ The immune modulating properties of HMOs, including interaction with dendritic cells and reduced antigen-antibody complex-induced chemokine release, are considered of critical importance for CMPA in terms of attenuation of allergic symptoms as well as promotion of tolerance development. $9,78,90,91$

The proposed link between HMOs, tolerance induction and prevention of allergy is primarily based on the certain effects of HMOs on gut microbiota, intestinal mucosal barrier and immunomodulation. ${ }^{8,92}$ These include the prebiotic effects, effects related to pathogen binding, enhanced epithelial barrier integrity and local and systemic modification of immune responses via SCFAs produced during HMOs fermentation, stimulation of mucus production and epithelial tight junction integrity to support the

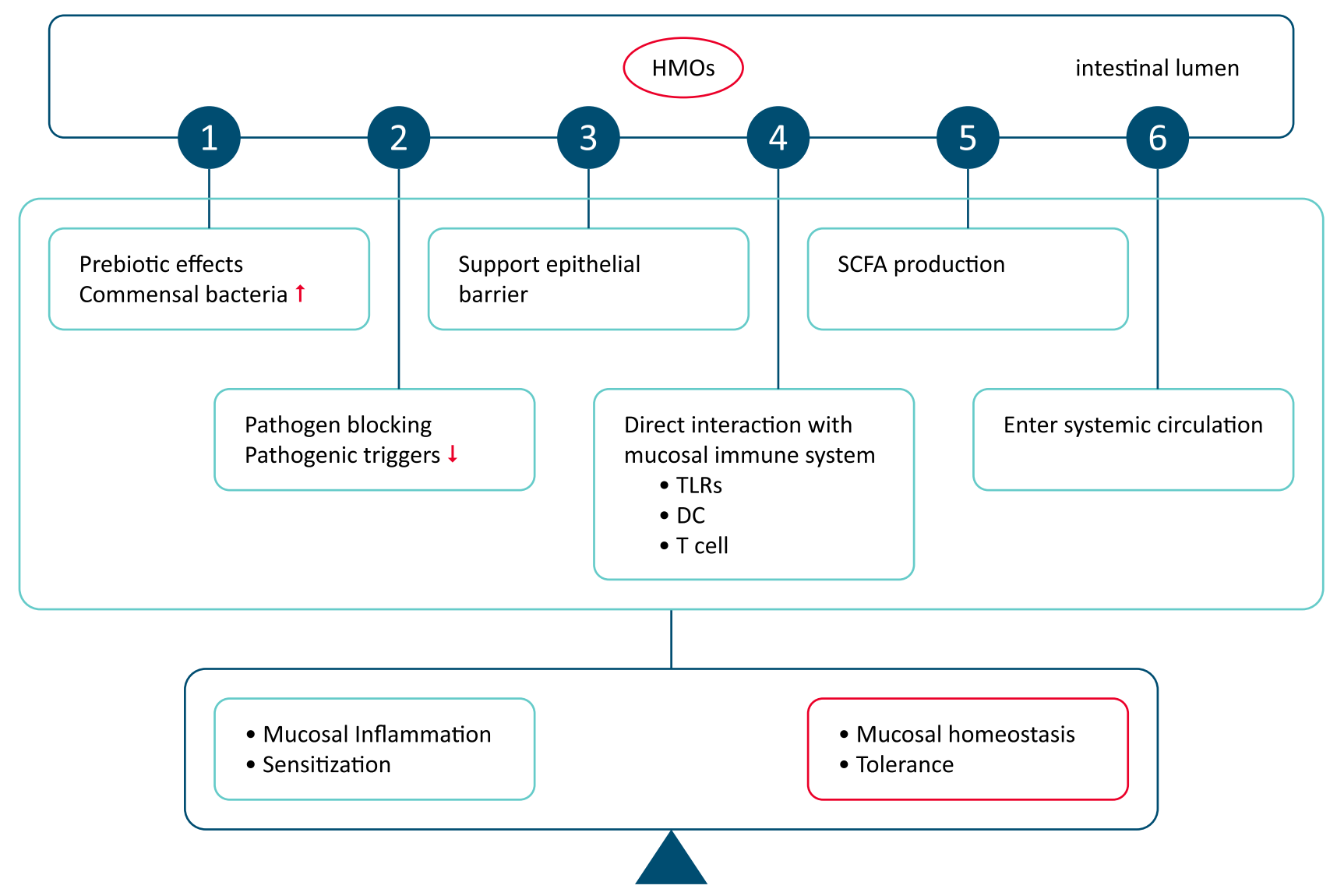

Figure 6 Potential roles of human milk oligosaccharide in enhanced tolerance induction and prevention of allergic diseases.

Notes: (I) Prebiotic effects and pathogen binding, enhanced epithelial barrier integrity and local and systemic modification of immune responses via SCFAs produced during HMOs fermentation. (2) Mucus production and epithelial tight junction integrity to support the physical barrier between the intestinal epithelium and the gut content. (3) Direct interaction with mucosal immune system (modulation of the response of DCs, protective mucosal immune development). (4) Immunomodulatory role beyond the gastrointestinal tract via entering systemic circulation through transportation over the intestinal epithelium. Adapted from Zuurveld M, van Witzenburg NP, Garssen J, et al. Immunomodulation by Human Milk Oligosaccharides: The Potential Role in Prevention of Allergic Diseases. Front Immunol. 2020;1 I:801. ${ }^{92 .}$

Abbreviation: HMO, human milk oligosaccharide. 
physical barrier between the intestinal epithelium and the gut content, the direct interaction with mucosal immune system (ie, modulation of the response of DCs, protective mucosal immune development) and finally the immunomodulatory role beyond the gastrointestinal tract via entering systemic circulation through transportation over the intestinal epithelium (Figure 6). ${ }^{92}$

\section{Gut microbiota, barrier integrity and prevention of allergy}

The composition and function of gut microbiota early in life is considered important in the development of food allergy ${ }^{93,94}$ Reduced gut microbial diversity and dysbiosis during early life is considered a risk factor for immunemediated diseases, such as allergy and asthma, through modulation of balance between effector or tolerant responses (activities of Th1 and Th2 cells) that leads to induction of a Th2-skewed immune response, with enhanced IgE responses to food antigens, and/or a defect in mounting proper regulatory T-cell responses. ${ }^{95}$

Hence, the manipulation of the microbiota of the infant during pregnancy or after birth, when there is significant immune-plasticity has been suggested to have an impact on allergy prevention. ${ }^{95}$ HMO-dependent increase in the growth of Bifidobacterium induces healthy gut microbiota development and contributes to the barrier integrity, while the direct effects of HMOs on the intestinal cell response (ie, reducing the cell growth, inducing differentiation and apoptosis, stimulating SCFA production) are also important for intestinal epithelial cell maturation and a proper intestinal barrier function. ${ }^{7,8,66,67,69-71}$

Notably, the infants who are exclusively breastfed by secretor mothers vs those breastfed by non-secretor mothers were reported to have a more rapid establishment of a bifidobacteria-predominant microbiota as well as lesser likelihood of diarrhea development. ${ }^{7,61}$ The stool bacterial profile in infants fed by a formula supplemented with $2^{\prime}$-FL and LNnT was reported to be more similar to that of the breastfed infants rather than those fed by a formula not supplemented with prebiotics. ${ }^{7,17}$ The amount of 2-FL in human milk was reported to inversely correlate with the incidence of Campylobacter diarrhea and the severity of infection with $E$. coli in infants, ${ }^{53,96}$ while besides their effects on gut bacteria, HMOs have also been reported to affect viral pathogens (ie, norovirus, rotavirus and HIV). ${ }^{97}$

HMOs are suggested to decrease the risk of preterm mortality and morbidity via their modulatory effects on the gut microbiome to protect against infections (ie, necrotizing enterocolitis, candidiasis) and several immune-related diseases, ${ }^{6,7,98}$ while recent studies have also suggested a possible association between HMOs and atopic dermatitis, milk allergy or allergic sensitization in the infant. ${ }^{13,89,99,100}$

Although the exact mechanism behind the association of microbiota composition with allergy development remains unknown, a protective role for SCFA has been suggested in asthma, arthritis, and colitis models as well as in allergic airway inflammation. ${ }^{93}$

\section{Immunomodulation, immune maturation and allergy} prevention

Although many studies regarding the composition of HMOs have been published, there are few publications on the immune-modulatory effects of individual HMOs. ${ }^{5,84,101}$ Although anti-inflammatory and immune regulatory potential of HMOs has been suggested, the exact mechanism underlying the effect of the specific HMOs on the risk for allergy development remains unknown. $^{5,84,101}$ Nonetheless, proposed mechanisms underlying immunomodulatory properties of HMOs include the pathogen decoy capacity, the prebiotic effect, the modulatory effect on the SCFA production and thus improved barrier integrity and the direct immune modulatory functions. ${ }^{31}$

The use of HMOs has been considered to reduce the levels of cytokine proteins including monocyte chemoattractant protein $1 / 2$, IL-8, IL-6 and IL- $1 \beta$, whereas to increase the level of cytokines associated with tissue repair and homeostasis. ${ }^{15}$ The HMO-mediated induction of tDCs with ability to modulate T-cell responses is considered likely to be a potential target for prevention and treatment of autoimmune diabetes and allergic diseases early in life. $^{78,102}$

Accordingly, superiority of HMOs over non-human prebiotic oligosaccharides was indicated in systemic and gastrointestinal immunomodulation. ${ }^{7,103}$ The plasma concentration of inflammatory cytokines was reported to be significantly lower in the breastfed infants and infants fed with 2'-FL-supplemented formula when compared to those fed with GOS-supplemented formula. ${ }^{79}$

The impact of 2'-FL on reduction of food allergy related symptoms was suggested to occur via induction of interleukin-10+ T-regulatory cells and indirect stabilization of mast cells in a mouse model. ${ }^{91}$ Moreover, cord blood T-cells exposed to sialylated HMOs were reported to increase the number of interferon- $\gamma$-producing CD3 $+\mathrm{CD} 4+$ and $\mathrm{CD} 3+\mathrm{CD} 8+$ lymphocytes and interleukin-13 


\section{(IL-13)-producing $\quad \mathrm{CD} 3+\mathrm{CD} 8+\quad$ lymphocytes. ${ }^{104}$} Sialylated HMOs were also reported to reduce IL-4 production in a subset of lymphocytes from adult patients with peanut allergy, indicating that certain sialylated HMOs may contribute to allergy prevention. ${ }^{55}$ Acidic HMOs were also reported to induce IFN-gamma and IL10 in human cord blood T cells, and to decrease IL-4 production in allergen-specific $\mathrm{T}$ cells. ${ }^{55}$ Supplementation of 2'-FL alone was reported to lower levels of TNFa, IL-1a, IL-1b, and IL-6, resembling those identified in breast fed infants. ${ }^{79}$

Hence, HMOs are considered to promote a shift in T-cell response toward a more balanced Th1/Th2cytokine production and low-level immunity, ${ }^{11}$ while inflammatory cytokine expression patterns of infants fed with HMO-supplemented formula and exclusively breastfed infants are considered to be similar. ${ }^{5}$

In a study of 266 infants followed for 5 years, infants born to secretor mothers were reported to have lower incidence of IgE-mediated eczema at 2 years, while authors also noted that 2'-FL was significantly associated with a lower incidence of any (acute or delayed) allergic disease in infants born with C-section. ${ }^{89}$ In addition, 2-year-old children who were born through $\mathrm{C}$-section and fed on an infant formula supplemented with 2'-FL were reported to have a lower risk of developing IgE-associated allergies compared to those fed unsupplemented formula. ${ }^{105}$

In a past study in the LIFE Child cohort, authors suggested possible indirect influences of allergic sensitization in the mother (based on IgE level) on HMO in human milk (indirectly via TGF beta2 and $\operatorname{IgA}$ ) along with a dependency between human milk IgA and specific HMOs (6'SL in particular). ${ }^{100}$

Nonetheless, it remains unclear whether specific HMO are more protective against allergy than other components, ${ }^{100}$ while in a recent study overall HMO profiles were reported to differ significantly between sensitized vs non-sensitized infants, whereas no positive or negative association was noted between sensitization and individual or total HMO. ${ }^{99}$

The exact role of the diverse HMO structures in inducing microbiome and immune development and providing protection against infections also remains unclarified. ${ }^{8}$ Indeed, the onset of IgE-associated allergic manifestations has recently been suggested to be linked to FUT2dependent oligosaccharide composition in human milk among infants with a high hereditary risk for allergies and those born by $\mathrm{C}$-section. ${ }^{31,89}$
Owing to growing evidence on the composition and effects of HMOs and the progress in biotechnology in the last years, there is increased availability of infant formulas comprising HMOs added to the complex mixture of prebiotic oligosaccharides. ${ }^{31}$ However, available studies regarding the impact of HMOs on infection and immune function are limited ${ }^{93}$ and most have focused on safety rather than on anti-infective or immunomodulatory profile. ${ }^{84,106}$ Hence, further clinical studies are needed to determine the full potential of HMOs within the early life immune development in order to clarify which HMO to be added to infant formula and the optimal strategies for concentration, composition and combination to prevent and treat allergy development in early and later life. . $^{8,31,78,93}$

\section{Potential utility of HMO-supplemented formula in infants and children with CMPA}

The IgE-mediated acute allergic reactions (wheezing, urticaria, vomiting, and anaphylaxis), non-IgE-mediated reactions, or mixed immune reactions can induce food allergy, while cow's milk and eggs are the most common food allergens in childhood food allergy. ${ }^{107-112}$

Overall, the management of CMPA is based on three intervention strategies including primary prevention of initial IgE sensitization, secondary prevention of the triggering of allergic reactions in IgE-sensitized children and tertiary prevention to reduce the expression of end-organ allergic disease in case of known food allergy via avoidance of allergic food and induction of tolerance. ${ }^{10,113-116}$

Although the benefits of human milk has consistently been reported in several areas (ie decreased risk of infections, overweightness and diabetes and increased cognitive functions), there is conflicting evidence regarding its role in the prevention of allergy. ${ }^{95,117}$

Past studies, mainly focused on IgE mediated CMPA, indicated that the incidence of CMPA in the first year of life is significantly lower in exclusively breastfed infants than in those fed by cow's milk based infant formula with intact protein $(<0.5$ vs $2-7.5 \%){ }^{95}$ Hence, it has been suggested that modifying human milk composition by selected interventions may enable more suitable human milk with the capacity to combat allergy epidemics in addition to infectious disease in offspring. ${ }^{95,118}$

HMOs are considered likely to modulate the susceptibility to both $\operatorname{IgE}$ and non-IgE mediated allergy in this regard, by affecting the immune development, gut inflammation and 
microbiota of the offspring, through indirect (prebiotics for gut microbiota) and direct systemic immunomodulatory effects (reduced antigen-antibody complex-induced chemokine release, improved Th1/Th2 balance). ${ }^{90,119,120}$

The likelihood of HMO to attenuate allergic symptoms has been reported in a mouse CMPA model study, ${ }^{91}$ while preclinical experiments indicated that HMOs are likely to induce tolerance development via interaction with human dendritic cells. $^{78}$

In a past study in breastfed infants with and without CMPA, the authors reported that all mothers with an infant with delayed-onset CMPA were reported to be secretors (active FUT2, milk containing 2'FL and LNFP I), whereas those with an infant with immediate-type (IgE-mediated) CMPA were non-secretor (FUT2 negative) mothers. ${ }^{13}$

Data from the first hypoallergenicity study by NowakWegrzyn et al related to use of an HMO-supplemented eHF in CMPA have confirmed the hypoallergenicity of the HMO-supplemented eHF and indicated the presence of the comparable levels of $2^{\prime} \mathrm{FL}$ and LNnT in the eHF and in the human milk. ${ }^{9}$

Authors also noted that while HMO are manufactured by bio-fermentation from lactose, there is no risk of residual milk allergen contamination due to removal of potential residual proteins via several purification steps. ${ }^{9}$ In fact, given that highly purified lactose is another microbiomemodifying substrate, authors also have hypothesized that the concomitant presence of lactose and HMO in the eHF may offer beneficial synergistic effects to the infants with CMPA in terms of developing gut microbiome and immune system. ${ }^{9,121,122}$ Hence, authors suggested the supplementation of eHF with $2^{\prime} \mathrm{FL}$ to be a safe strategy in the management CMPA, not increasing the risk of clinical reactions compared to the non-HMO control formula. ${ }^{9}$

Nonetheless, the potential utility of HMOsupplemented formula in the management of CMPA still needs further exploration, particularly in terms of their potential to accelerate tolerance development or to reduce the frequency of infections in infants and children with CMPA. ${ }^{15,123}$

\section{Conclusions}

This review by pediatric gastroenterology and allergyimmunology experts emphasize the marked contribution of HMOs to the beneficial effects of human milk in early life in terms of provision of healthy neonatal microbial colonization, fine-tuning of inflammatory processes and immune defense and maturation. The participating experts consider HMOs amongst the most critical bioactive components of human milk, given that they act as antimicrobials and antivirals by preventing pathogen adhesion to epithelial cells, as intestinal epithelial cell modulators by enhancing maturation of intestinal mucosa and intestinal epithelial barrier function, as prebiotics and effectors of the gut microbiota by selectively enriching gut bacteria and thereby promoting healthy microbiota composition and as immunomodulators by modulating immune cells indirectly via changing the infant gut microbiota composition, and directly via affecting immune cells on a systemic level. The potential advantages of HMO-supplemented formula over GOS and FOS supplemented formulas are considered to be ability of HMOs to form the preferred substrate for bifidobacteria and suppress potential gut pathogens, to positively influence gut epithelial integrity, apoptosis, and intestinal permeability and to have indirect and direct immunomodulatory properties.

The participating experts emphasize that reduced gut microbial diversity and dysbiosis during early life as well as induction of a Th2-skewed immune response are considered a risk factor for immune-mediated diseases, such as allergy and asthma in early life. Accordingly, given the marked impact of HMOs on development of healthy gut microbiota and indirect (pathogen decoy capacity, the prebiotic effect, the stimulatory effects on SCFA production and tDCs) and direct anti-inflammatory and immune modulatory functions that promote a shift in T-cell response toward a more balanced Th1/Th2-cytokine production, the participating experts consider the proposed link between HMOs and prevention of allergy to be primarily based on the effects of HMO on gut microbiota, intestinal mucosal barrier and immunomodulation.

The increased availability of HMO from commercial sources as well as accumulating evidence on safety and tolerability of HMO-supplemented formulas seem important given that they seem to be promising alternatives in the management of infants and children with CMPA due to beneficial effects on gut microbiota, intestinal mucosal barrier and immunomodulation as well as anti-inflammatory and anti-infective properties. Accordingly, representing the most critical bioactive component of human milk, responsible for gastrointestinal and immune benefits, HMO-supplemented formulas seem to have potential for provision of infants with CMPA with a formula that brings the similar health benefits with the human milk. Nonetheless, the effects of individual as well as complex mixtures of $\mathrm{HMO}$ in relation to well-defined clinical 
and immune outcomes and tolerance development needs to be further explored to fully realize the immunomodulatory mechanisms and the potential for HMO in prevention of allergic disease development and in the management of CMPA. This review is an expert panel summary report addressing the biological roles of HMOs and their beneficial effects in early life with special emphasis on CMPA to increase awareness about CMPA and to achieve a consensus amongst the different specialties in the CMPA patient care via panel discussions regarding the unmet needs and controversial topics. Hence, focusing on not only the overall beneficial effects of HMOs in early life but also specifically addressing the utility of HMO-supplemented formula in the management of CMPA and in the development of tolerance in the early life with potential long-term positive impacts, this paper provides the opinion and perspectives of the medical community and may help to inform the design of future HMO studies. The expert panel suggests the long-term effects of different HMOs on development of tolerance in early life, their effects on gut microbiota, intestinal mucosal barrier, immunomodulation and immune maturation are the relevant clinical aspects to address when investigating the role of HMOs in prevention of allergy and in reducing the severity of allergy.

\section{Acknowledgments}

The authors would like to thank Cagla Ayhan, MD and Prof. Sule Oktay, MD, PhD from KAPPA Consultancy Training Research Ltd, Istanbul who provided editorial support funded by Abbott Nutrition Turkey.

\section{Author Contributions}

All authors made a significant contribution to the work reported, whether that is in the conception, study design, execution, acquisition of data, analysis and interpretation, or in all these areas; took part in drafting, revising or critically reviewing the article; gave final approval of the version to be published; have agreed on the journal to which the article has been submitted; and agree to be accountable for all aspects of the work.

\section{Funding}

This study was supported by Abbott Nutrition Turkey which played a role in organization of expert panel meeting and compensation for editorial support. Abbott Nutrition Turkey had no role in study design, data collection and analysis, decision to publish, or preparation of the manuscript.

\section{Disclosure}

Bulent Enis Sekerel reports speaker fees from Abbott, Abdi Ibrahim, and Sandoz, outside the submitted work. Aydan Kansu reports speaker fees from Abbott, outside the submitted work. The authors declare that they have no other conflicts of interest in this work.

\section{References}

1. World Health Organization. Maternal, Newborn, Child and Adolescent Health. Geneva: World Health Organization; 2017. Available from: http://www.who.int/maternal_child_adolescent/ topics/child/nutrition/breastfeeding/en/. Accessed January 4, 2021..

2. Agostoni C, Braegger C, Decsi T, et al; ESPGHAN Committee on Nutrition. Breast-feeding: a commentary by the ESPGHAN Committee on Nutrition. J Pediatr Gastroenterol Nutr. 2009;49 (1):112-125. doi:10.1097/MPG.0b013e31819f1e05

3. Bode L. Recent advances on structure, metabolism, and function of human milk oligosaccharides. J Nutr. 2006;136(8):2127-2130. doi:10.1093/jn/136.8.2127

4. Vandenplas Y, Berger B, Carnielli VP, et al. Human milk oligosaccharides: 2'-Fucosyllactose (2'-FL) and Lacto-N-Neotetraose (LNnT) in infant formula. Nutrients. 2018;10(9):1161. doi:10.3390/nu10091161

5. Plaza-Díaz J, Fontana L, Gil A. Human milk oligosaccharides and immune system development. Nutrients. 2018;10(8):1038. doi: $10.3390 /$ nu10081038

6. Akkerman R, Faas MM, de Vos P. Non-digestible carbohydrates in infant formula as substitution for human milk oligosaccharide functions: effects on microbiota and gut maturation. Crit Rev Food Sci Nutr. 2019;59(9):1486-1497. doi:10.1080/10408398.2017.1414030

7. Hegar B, Wibowo Y, Basrowi RW, et al. The role of two human milk oligosaccharides, 2'-fucosyllactose and Lacto-N-Neotetraose, in infant nutrition. Pediatr Gastroenterol Hepatol Nutr. 2019;22 (4):330-340. doi:10.5223/pghn.2019.22.4.330

8. Ayechu-Muruzabal V, van Stigt AH, Mank M, et al. Diversity of human milk oligosaccharides and effects on early life immune development. Front Pediatr. 2018;6:239. doi:10.3389/ fped.2018.00239

9. Nowak-Wegrzyn A, Czerkies L, Reyes K, Collins B, Heine RG. Confirmed hypoallergenicity of a novel whey-based extensively hydrolyzed infant formula containing two human milk oligosaccharides. Nutrients. 2019;11(7):1447. doi:10.3390/ nu11071447

10. Donovan SM, Comstock SS. Human milk oligosaccharides influence neonatal mucosal and systemic immunity. Ann Nutr Metab. 2016;69 Suppl 2(Supp12):42-51.

11. Bode L. Human milk oligosaccharides: every baby needs a sugar mama. Glycobiology. 2012;22(9):1147-1162. doi:10.1093/glycob/ cws074

12. Azad MB, Konya T, Guttman DS, et al. Infant gut microbiota and food sensitization: associations in the first year of life. Clin Exp Allergy. 2015;45(3):632-643. doi:10.1111/cea.12487

13. Seppo AE, Autran CA, Bode L, Järvinen KM. Human milk oligosaccharides and development of cow's milk allergy in infants. $J$ Allergy Clin Immunol. 2017;139(2):708-711. doi:10.1016/j.jaci.2016.08.031

14. Gibson GR, Wang X. Regulatory effects of bifidobacteria on the growth of other colonic bacteria. J Appl Bacteriol. 1994;77 (4):412-420. doi:10.1111/j.1365-2672.1994.tb03443.x

15. Wiciński M, Sawicka E, Gębalski J, Kubiak K, Malinowski B. Human milk oligosaccharides: health benefits, potential applications in infant formulas, and pharmacology. Nutrients. 2020;12 (1):266. doi:10.3390/nu12010266 
16. Harmsen HJ, Wildeboer-Veloo AC, Raangs GC, et al. Analysis of intestinal flora development in breast-fed and formula-fed infants by using molecular identification and detection methods. J Pediatr Gastroenterol Nutr. 2000;30(1):61-67. doi:10.1097/ 00005176-200001000-00019

17. Puccio G, Alliet P, Cajozzo C, et al. Effects of infant formula with human milk oligosaccharides on growth and morbidity: a randomized multicenter trial. $J$ Pediatr Gastroenterol Nutr. 2017;64(4):624-631. doi:10.1097/MPG.0000000000001520

18. Aly E, Darwish AA, Lopez-Nicolas R, Frontela-Saseta C, RosBerruezo G. Bioactive components of human milk: similarities and differences between human milk and infant formula, selected topics in breastfeeding, R. Mauricio Barría P. IntechOpen. 2018. Available from: https://www.intechopen.com/chapters/58668. Accessed August 27, 2021.

19. Ballard O, Morrow AL. Human milk composition: nutrients and bioactive factors. Pediatr Clin North Am. 2013;60(1):49-74. doi:10.1016/j.pcl.2012.10.002

20. Levy O. Innate immunity of the newborn: basic mechanisms and clinical correlates. Nat Rev Immunol. 2007;7(5):379-390. doi:10.1038/nri2075

21. Newburg DS, He Y. Neonatal gut microbiota and human milk glycans cooperate to attenuate infection and inflammation. Clin Obstet Gynecol. 2015;58(4):814-826. doi:10.1097/GRF.0000000 000000156

22. Kulinich A, Liu L. Human milk oligosaccharides: the role in the fine-tuning of innate immune responses. Carbohydr Res. 2016;432:62-70. doi:10.1016/j.carres.2016.07.009

23. Lloyd-Price J, Abu-Ali G, Huttenhower C. The healthy human microbiome. Genome Med. 2016;8(1):51. doi:10.1186/s13073016-0307-y

24. Turfkruyer M, Verhasselt V. Breast milk and its impact on maturation of the neonatal immune system. Curr Opin Infect Dis. 2015;28(3):199-206. doi:10.1097/QCO.0000000000000165

25. Cacho NT, Lawrence RM. Innate immunity and breast milk. Front Immunol. 2017;8:584. doi:10.3389/fimmu.2017.00584

26. Futata EA, Fusaro AE, de Brito CA, Sato MN. The neonatal immune system: immunomodulation of infections in early life. Expert Rev Anti Infect Ther. 2012;10(3):289-298. doi:10.1586/ eri.12.9

27. Renz H, Holt PG, Inouye M, Logan AC, Prescott SL, Sly PD. An exposome perspective: early-life events and immune development in a changing world. J Allergy Clin Immunol. 2017;140(1):24-40. doi:10.1016/j.jaci.2017.05.015

28. Eidelman AI. Breastfeeding and the use of human milk: an analysis of the American Academy of Pediatrics 2012 breastfeeding policy statement. Breastfeed Med. 2012;7(5):323-324. doi:10.1089/bfm.2012.0067

29. Oddy WH. Breastfeeding, childhood asthma, and allergic disease. Ann Nutr Metab. 2017;70(Suppl 2):26-36. doi:10.1159/000457920

30. Vieira Borba V, Sharif K, Shoenfeld Y. Breastfeeding and autoimmunity: programing health from the beginning. Am J Reprod Immunol. 2018;79:1. doi:10.1111/aji.12778

31. Munblit D, Peroni DG, Boix-Amorós A, et al. Human milk and allergic diseases: an unsolved puzzle. Nutrients. 2017;9(8):894. doi:10.3390/nu9080894

32. Akobeng AK, Ramanan AV, Buchan I, Heller RF. Effect of breast feeding on risk of coeliac disease: a systematic review and meta-analysis of observational studies. Arch Dis Child. 2006;91 (1):39-43. doi:10.1136/adc.2005.082016

33. Perkin MR, Logan K, Tseng A, et al. Randomized trial of introduction of allergenic foods in breast-fed infants. $N$ Engl $J$ Med. 2016;374(18):1733-1743. doi:10.1056/NEJMoa1514210

34. WHO. Global Strategy for Infant and Young Child Feeding, the Optimal Duration of Exclusive Breastfeeding. Geneva, Switzerland: World Health Organization; 2001.
35. Lönnerdal B, Hernell O. An opinion on "staging" of infant formula- a developmental perspective on infant feeding. J Pediatr Gastroenterol Nutr. 2016;62(1):9-21. doi:10.1097/MPG.00000 00000000806

36. Martin CR, Ling PR, Blackburn GL. Review of infant feeding: key features of breast milk and infant formula. Nutrients. 2016;8 (5):279. doi:10.3390/nu8050279

37. Boehm G, Stahl B. Oligosaccharides from milk. J Nutr. 2007;137 (3Suppl 2):847S-849S. doi:10.1093/jn/137.3.847S

38. Thurl S, Munzert M, Henker J, et al. Variation of human milk oligosaccharides in relation to milk groups and lactational periods. Br J Nutr. 2010;104(9):1261-1271. doi:10.1017/S000 7114510002072

39. McGuire MK, Meehan CL, McGuire MA, et al. What's normal? Oligosaccharide concentrations and profiles in milk produced by healthy women vary geographically. Am J Clin Nutr. 2017;105 (5):1086-1100. doi:10.3945/ajcn.116.139980

40. Thum C, Wall CR, Weiss GA, Wang W, Szeto IM, Day L. Changes in HMO concentrations throughout lactation: influencing factors, health effects and opportunities. Nutrients. 2021;13 (7):2272. doi:10.3390/nu13072272

41. Plows JF, Berger PK, Jones RB, et al. Longitudinal changes in Human Milk Oligosaccharides (HMOs) over the course of 24 months of lactation. J Nutr. 2021;151(4):876-882. doi:10.1093/ $\mathrm{jn} / \mathrm{nxaa} 427$

42. Siziba LP, Mank M, Stahl B, et al. Human milk oligosaccharide profiles over 12 months of lactation: the Ulm SPATZ Health Study. Nutrients. 2021;13(6):1973. doi:10.3390/nu13061973

43. Berger P, Plows J, Jones R, et al. Human milk oligosaccharides are stable over one-week of lactation and over six-hours following a standardized meal current developments in nutrition. Curr Dev Nutr. 2021;5(Supplement_2):719. doi:10.1093/cdn/nzab0 46_016

44. Chouraqui J-P. Does the contribution of human milk oligosaccharides to the beneficial effects of breast milk allow us to hope for an improvement in infant formulas? Crit Rev Food Sci Nutr. 2021;61:1503-1514. doi:10.1080/10408398.2020.1761772

45. Yu Y, Mishra S, Song X, et al. Functional glycomic analysis of human milk glycans reveals the presence of virus receptors and embryonic stem cell biomarkers. J Biol Chem. 2012;287 (53):44784-44799. doi:10.1074/jbc.M112.425819

46. Goehring KC, Kennedy AD, Prieto PA, Buck RH. Direct evidence for the presence of human milk oligosaccharides in the circulation of breastfed infants. PLoS One. 2014;9(7):e101692. doi:10.1371/journal.pone.0101692

47. Bode L. The functional biology of human milk oligosaccharides. Early Hum Dev. 2015;91(11):619-622. doi:10.1016/j.earlhumdev. 2015.09.001

48. Smilowitz JT, O’Sullivan A, Barile D, German JB, Lönnerdal B, Slupsky CM. The human milk metabolome reveals diverse oligosaccharide profiles. J Nutr. 2013;143(11):1709-1718. doi:10.39 45/jn. 113.178772

49. Xu G, Davis JC, Goonatilleke E, Smilowitz JT, German JB, Lebrilla CB. Absolute quantitation of human milk oligosaccharides reveals phenotypic variations during lactation. $J$ Nutr. 2017;147(1):117-124. doi:10.3945/jn.116.238279

50. Smilowitz JT, Lebrilla CB, Mills DA, German JB, Freeman SL. Breast milk oligosaccharides: structure-function relationships in the neonate. Annu Rev Nutr. 2014;34:143-169. doi:10.1146/ annurev-nutr-071813-105721

51. Bode L, Jantscher-Krenn E. Structure-function relationships of human milk oligosaccharides. Adv Nutr. 2012;3(3):383S-391S. doi:10.3945/an.111.001404

52. Lewis ZT, Totten SM, Smilowitz JT, et al. Maternal fucosyltransferase 2 status affects the gut bifidobacterial communities of breastfed infants. Microbiome. 2015;3:13. doi:10.1186/s40168-015-0071-z 
53. Newburg DS, Ruiz-Palacios GM, Altaye M, et al. Innate protection conferred by fucosylated oligosaccharides of human milk against diarrhea in breastfed infants. Glycobiology. 2004;14 (3):253-263. doi:10.1093/glycob/cwh020

54. Rudloff S, Pohlentz G, Borsch C, Lentze MJ, Kunz C. Urinary excretion of in vivo ${ }^{13} \mathrm{C}$-labelled milk oligosaccharides in breastfed infants. Br J Nutr. 2012;107(7):957-963. doi:10.1017/ S0007114511004016

55. Eiwegger T, Stahl B, Haidl P, et al. Prebiotic oligosaccharides: in vitro evidence for gastrointestinal epithelial transfer and immunomodulatory properties. Pediatr Allergy Immunol. 2010;21 (8):1179-1188. doi:10.1111/j.1399-3038.2010.01062.x

56. Ackerman DL, Doster RS, Weitkamp JH, Aronoff DM, Gaddy JA, Townsend SD. Human milk oligosaccharides exhibit antimicrobial and antibiofilm properties against Group B Streptococcus. ACS Infect Dis. 2017;3(8):595-605. doi:10.1021/ acsinfecdis.7b00064

57. Moore RE, Xu LL, Townsend SD. Prospecting human milk oligosaccharides as a defense against viral infections. ACS Infect Dis. 2021;7(2):254-263. doi:10.1021/acsinfecdis.0c00807

58. Craft KM, Townsend SD. Mother knows best: deciphering the antibacterial properties of human milk oligosaccharides. Acc Chem Res. 2019;52(3):760-768. doi:10.1021/acs.accounts.8b0 0630

59. Ruiz-Palacios GM, Cervantes LE, Ramos P, Chavez-Munguia B, Newburg DS. Campylobacter jejuni binds intestinal $\mathrm{H}(\mathrm{O})$ antigen (Fuc alpha 1, 2Gal beta 1, 4GlcNAc), and fucosyloligosaccharides of human milk inhibit its binding and infection. $J$ Biol Chem. 2003;278:14112-14120. doi:10.1074/jbc.M207744200

60. Ida"npa"a"n-Heikkila" I, Simon PM, Zopf D, et al. Oligosaccharides interfere with the establishment and progression of experimental pneumococcal pneumonia. $J$ Infect Dis. 1997;176:704-712. doi:10.1086/514094

61. Morrow AL, Ruiz-Palacios GM, Altaye M, et al. Human milk oligosaccharides are associated with protection against diarrhea in breast-fed infants. J Pediatr. 2004;145(3):297-303. doi:10.1016/j. jpeds.2004.04.054

62. Lin AE, Autran CA, Szyszka A, et al. Human milk oligosaccharides inhibit growth of group B Streptococcus. J Biol Chem. 2017;292(27):11243e9. doi:10.1074/jbc.M117.789974

63. Cheng Y-J, Yeung C-Y. Recent advance in infant nutrition: human milk oligosaccharides. Pediatr Neonatol. 2021;62(4):347-353. doi:10.1016/j.pedneo.2020.12.013

64. Nolan LS, Rimer JM, Good M. The role of human milk oligosaccharides and probiotics on the neonatal microbiome and risk of necrotizing enterocolitis: a narrative review. Nutrients. 2020;12 (10):3052. doi:10.3390/nu12103052

65. Craft KM, Gaddy JA, Townsend SD. Human Milk Oligosaccharides (HMOs) sensitize group B Streptococcus to clindamycin, erythromycin, gentamicin, and minocycline on a strain specific basis. ACS Chem Biol. 2018;13(8):2020-2026. doi:10.1021/acschembio.8b00661

66. Chen X. Human Milk Oligosaccharides (HMOS): structure, function, and enzyme-catalyzed synthesis. Adv Carbohydr Chem Biochem. 2015;72:113-190.

67. Kuntz S, Kunz C, Rudloff S. Oligosaccharides from human milk induce growth arrest via $\mathrm{G} 2 / \mathrm{M}$ by influencing growth-related cell cycle genes in intestinal epithelial cells. Br J Nutr. 2009;101 (9):1306-1315. doi:10.1017/S0007114508079622

68. Angeloni S, Ridet JL, Kusy N, et al. Glycoprofiling with micro-arrays of glycoconjugates and lectins. Glycobiology. 2005;15(1):31-41. doi:10.1093/glycob/cwh143

69. Asakuma S, Hatakeyama E, Urashima T, et al. Physiology of consumption of human milk oligosaccharides by infant gut-associated bifidobacteria. $J$ Biol Chem. 2011;286 (40):34583-34592. doi:10.1074/jbc.M111.248138
70. Plöger S, Stumpff F, Penner GB, et al. Microbial butyrate and its role for barrier function in the gastrointestinal tract. Ann N Y Acad Sci. 2012;1258:52-59. doi:10.1111/j.1749-6632.2012.06553.x

71. Reichardt N, Duncan SH, Young P, et al. Phylogenetic distribution of three pathways for propionate production within the human gut microbiota. ISME J. 2014;8(6):1323-1335. doi:10.1038/ismej.2014.14

72. Holscher HD, Davis SR, Tappenden KA. Human milk oligosaccharides influence maturation of human intestinal Caco-2Bbe and HT-29 cell lines. J Nutr. 2014;144(5):586-591. doi:10.3945/ jn.113.189704

73. Garrido D, Kim JH, German JB, Raybould HE, Mills DA. Oligosaccharide binding proteins from bifidobacterium longum subsp. infantis reveal a preference for host glycans. PLoS One. 2011;6(3):e17315. doi:10.1371/journal.pone.0017315

74. De Leoz ML, Kalanetra KM, Bokulich NA, et al. Human milk glycomics and gut microbial genomics in infant feces show a correlation between human milk oligosaccharides and gut microbiota: a proof-of-concept study. J Proteome Res. 2015;14 (1):491-502. doi:10.1021/pr500759e

75. Marcobal A, Sonnenburg JL. Human milk oligosaccharide consumption by intestinal microbiota. Clin Microbiol Infect. 2012;18 (suppl 4):12-15. doi:10.1111/j.1469-0691.2012.03863.x

76. Rautava S, Luoto R, Salminen S, Isolauri E. Microbial contact during pregnancy, intestinal colonization and human disease. Nat Rev Gastroenterol Hepatol. 2012;9(10):565-576. doi:10.1038/ nrgastro.2012.144

77. Simon AK, Hollander GA, McMichael A. Evolution of the immune system in humans from infancy to old age. Proc Biol Sci. 2015;282(1821):20143085.

78. Xiao L, van De Worp WR, Stassen R, et al. Human milk oligosaccharides promote immune tolerance via direct interactions with human dendritic cells. Eur J Immunol. 2019;49 (7):1001-1014. doi:10.1002/eji.201847971

79. Goehring KC, Marriage BJ, Oliver JS, Wilder JA, Barrett EG, Buck RH. Similar to those who are breastfed, infants fed a formula containing 2'-fucosyllactose have lower inflammatory cytokines in a randomized controlled trial. J Nutr. 2016;146 (12):2559-2566. doi:10.3945/jn.116.236919

80. Reverri EJ, Devitt AA, Kajzer JA, Baggs GE, Borschel MW. Review of the clinical experiences of feeding infants formula containing the human milk oligosaccharide 2 '-fucosyllactose. Nutrients. 2018;10(10):1346. doi:10.3390/nu10101346

81. Kunz C, Rudloff S, Baier W, Klein N, Strobel S. Oligosaccharides in human milk: structural, functional, and metabolic aspects. Annu Rev Nutr. 2000;20:699-722. doi:10.1146/annurev.nutr.20.1.699

82. Vandenplas Y, Zakharova I, Dmitrieva Y. Oligosaccharides in infant formula: more evidence to validate the role of prebiotics. $\mathrm{Br} J$ Nutr. 2015;113(9):1339-1344. doi:10.1017/S0007114515000823

83. Petschacher B, Nidetzky B. Biotechnological production of fucosylated human milk oligosaccharides: prokaryotic fucosyltransferases and their use in biocatalytic cascades or whole cell conversion systems. J Biotechnol. 2016;235:61-83. doi:10.1016/ j.jbiotec.2016.03.052

84. Marriage BJ, Buck RH, Goehring KC, Oliver JS, Williams JA. Infants fed a lower calorie formula with $2^{\prime} \mathrm{FL}$ show growth and 2'FL uptake like breast-fed infants. J Pediatr Gastroenterol Nutr. 2015;61(6):649-658. doi:10.1097/MPG.0000000000000889

85. Matsuki T, Yahagi $\mathrm{K}$, Mori $\mathrm{H}$, et al. A key genetic factor for fucosyllactose utilization affects infant gut microbiota development. Nat Commun. 2016;24(7):11939. doi:10.1038/ ncomms 11939

86. Smith-Brown P, Morrison M, Krause L, Davies PSW, Weir TL. Mothers secretor status affects development of childrens microbiota composition and function: a Pilot Study. PLoS One. 2016;11 (9):e0161211. doi:10.1371/journal.pone.0161211 
87. Román E, Moreno Villares JM, Domínguez Ortega F, et al. Realworld study in infants fed with an infant formula with two human milk oligosaccharides. Nutr Hosp. 2020;37(4):698-706.

88. Kajzer J, Oliver J, Marriage B. Gastrointestinal tolerance of formula supplemented with oligosaccharides. FASEB J. 2016;30:671.

89. Sprenger N, Odenwald H, Kukkonen AK, Kuitunen M, Savilahti E, Kunz C. FUT2-dependent breast milk oligosaccharides and allergy at 2 and 5 years of age in infants with high hereditary allergy risk. Eur J Nutr. 2017;56(3):1293-1301. doi:10.1007/s00394-016-1180-6

90. Zehra S, Khambati I, Vierhout M, Mian MF, Buck R, Forsythe P. Human milk oligosaccharides attenuate antigen-antibody complex induced chemokine release from human intestinal epithelial cell lines. J Food Sci. 2018;83(2):499-508. doi:10.1111/1750-3841.14039

91. Castillo-Courtade L, Han S, Lee S, Mian FM, Buck R, Forsythe P. Attenuation of food allergy symptoms following treatment with human milk oligosaccharides in a mouse model. Allergy. 2015;70 (9):1091-1102. doi:10.1111/all.12650

92. Zuurveld $M$, van Witzenburg NP, Garssen J, et al. Immunomodulation by human milk oligosaccharides: the potential role in prevention of allergic diseases. Front Immunol. 2020;11:801. doi:10.3389/fimmu.2020.00801

93. Triantis V, Bode L, van Neerven RJJ. Immunological effects of human milk oligosaccharides. Front Pediatr. 2018;6:190. doi:10.3389/fped.2018.00190

94. Aitoro R, Paparo L, Amoroso A, et al. Gut microbiota as a target for preventive and therapeutic intervention against food allergy. Nutrients. 2017;9(7):672. doi:10.3390/nu9070672

95. Vandenplas Y, Meyer R, Chouraqui JP, et al. The role of milk feeds and other dietary supplementary interventions in preventing allergic disease in infants: fact or fiction? Clin Nutr. 2021;40 (2):358-371. doi:10.1016/j.clnu.2020.10.025

96. Morrow AL, Ruiz-Palacios GM, Altaye M, et al. Human milk oligosaccharide blood group epitopes and innate immune protection against campylobacter and calicivirus diarrhea in breastfed infants. Adv Exp Med Biol. 2004;554:443-446.

97. Etzold S, Bode L. Glycan-dependent viral infection in infants and the role of human milk oligosaccharides. Curr Opin Virol. 2014;7:101-107. doi:10.1016/j.coviro.2014.06.005

98. Moukarzel S, Bode L. Human milk oligosaccharides and the preterm infant: a journey in sickness and in health. Clin Perinatol. 2017;44(1):193-207. doi:10.1016/j.clp.2016.11.014

99. Miliku K, Robertson B, Sharma AK, et al. Human milk oligosaccharide profiles and food sensitization among infants in the CHILD Study. Allergy. 2018;73(10):2070-2073. doi:10.1111/all.13476

100. Michel L, Shevlyakova M, Ní Cléirigh E, et al. Novel approach to visualize the inter-dependencies between maternal sensitization, breast milk immune components and human milk oligosaccharides in the LIFE child cohort. PLoS One. 2020;15(4):e0230472. doi:10.1371/journal.pone.0230472

101. Doherty AM, Lodge CJ, Dharmage SC, Dai X, Bode L, Lowe AJ. Human milk oligosaccharides and associations with immune-mediated disease and infection in childhood: a systematic review. Front Pediatr. 2018;6:91. doi:10.3389/ fped.2018.00091

102. Xiao L, Van't Land B, van de Worp WRPH, Stahl B, Folkerts G, Garssen J. Early-life nutritional factors and mucosal immunity in the development of autoimmune diabetes. Front Immunol. 2017;8:1219. doi:10.3389/fimmu.2017.01219

103. Comstock SS, Li M, Wang M, et al. Dietary human milk oligosaccharides but not prebiotic oligosaccharides increase circulating natural killer cell and mesenteric lymph node memory $\mathrm{T}$ cell populations in noninfected and rotavirus-infected neonatal piglets. J Nutr. 2017;147(6):1041-1047. doi:10.3945/jn.116.24 3774
104. Eiwegger T, Stahl B, Schmitt J, et al. Human milk-derived oligosaccharides and plant-derived oligosaccharides stimulate cytokine production of cord blood T-cells in vitro. Pediatr Res. 2004;56(4):536-540. doi:10.1203/01.PDR.0000139411. 35619.B4

105. Sprenger N, Lee LY, De Castro CA, Steenhout P, Thakkar SK, Wiley AS. Longitudinal change of selected human milk oligosaccharides and association to infants' growth, an observatory, single center, longitudinal cohort study. PLoS One. 2017;12(2): e0171814. doi:10.1371/journal.pone.0171814

106. Meli F, Puccio G, Cajozzo C, et al. Growth and safety evaluation of infant formulae containing oligosaccharides derived from bovine milk: a randomized, double-blind, noninferiority trial. BMC Pediatr. 2014;14(1):306. doi:10.1186/s12887-014-0306-3

107. Lee S. IgE-mediated food allergies in children: prevalence, triggers, and management. Korean J Pediatr. 2017;60(4):99-105. doi:10.3345/kjp.2017.60.4.99

108. Sicherer SH, Sampson HA. Food allergy: epidemiology, pathogenesis, diagnosis, and treatment. $J$ Allergy Clin Immunol. 2014;133(2):291-307. doi:10.1016/j.jaci.2013.11.020

109. Rona RJ, Keil T, Summers C, et al. The prevalence of food allergy: a meta-analysis. J Allergy Clin Immunol. 2007;120 (3):638-646. doi:10.1016/j.jaci.2007.05.026

110. Fiocchi A, Brozek J, Schünemann H, et al. World Allergy Organization (WAO) Diagnosis and Rationale for Action against Cow's Milk Allergy (DRACMA) guidelines. World Allergy Organ J. 2010;3(4):57-161.

111. Kansu A, Yüce A, Dalgıç B, Şekerel BE, Çullu-çokuğraş F, Çokuğraş $H$. Consensus statement on diagnosis, treatment and follow-up of cow's milk protein allergy among infants and children in Turkey. Turk J Pediatr. 2016;58(1):1-11. doi:10.24953/ turkjped.2016.01.001

112. Kahveci M, Koken G, Şahiner ÜM, Soyer Ö, Şekerel BE. Immunoglobulin E-mediated food allergies differ in east Mediterranean children aged 0-2 years. Int Arch Allergy Immunol. 2020;181(5):365-374. doi:10.1159/000505996

113. Sackesen C, Altintas DU, Bingol A, et al. Current trends in tolerance induction in cow's milk allergy: from passive to proactive strategies. Front Pediatr. 2019;7:372. doi:10.3389/ fped.2019.00372

114. Du Toit G, Tsakok T, Lack S, Lack G. Prevention of food allergy. J Allergy Clin Immunol. 2016;137(4):998-1010. doi:10.1016/j. jaci.2016.02.005

115. Crittenden RG, Bennett LE. Cow's milk allergy: a complex disorder. J Am Coll Nutr. 2005;24(6 Suppl):582S-591S. doi:10.1080/07315724.2005.10719507

116. Yüce A, Dalgıç B, Çullu-çokuğraş F, et al. Cow`s milk protein allergy awareness and practice among Turkish pediatricians: a questionnaire-survey. Turk J Pediatr. 2017;59(3):233-243. doi:10.24953/turkjped.2017.03.002

117. Victora CG, Bahl R, Barros AJ, França GV, Horton S, Krasevec J. Breastfeeding in the 21st century: epidemiology, mechanisms, and lifelong effect. Lancet. 2016;387(10017):475-490. doi:10.1016/S0140-6736(15)01024-7

118. Munblit D, Verhasselt V. Allergy prevention by breastfeeding: possible mechanisms and evidence from human cohorts. Curr Opin Allergy Clin Immunol. 2016;16(5):427-433. doi:10.1097/ ACI.0000000000000303

119. D'Auria E, Salvatore S, Pozzi E, et al. Cow's milk allergy: immunomodulation by dietary intervention. Nutrients. 2019;11 (6):1399. doi:10.3390/nu11061399

120. Dzidic M, Mira A, Artacho A, Abrahamsson TR, Jenmalm MC, Collado MC. Allergy development is associated with consumption of breastmilk with a reduced microbial richness in the first month of life. Pediatr Allergy Immunol. 2020;31(3):250-257. doi:10.1111/pai.13176 
121. Thompson-Chagoyan OC, Fallani M, Maldonado J, et al. Faecal microbiota and short-chain fatty acid levels in faeces from infants with cow's milk protein allergy. Int Arch Allergy Immunol. 2011;156(3):325-332. doi:10.1159/000323893

122. Berni Canani R, De Filippis F, Nocerino R, et al. Gut microbiota composition and butyrate production in children affected by nonIgE-mediated cow's milk allergy. Sci Rep. 2018;8(1):12500. doi:10.1038/s41598-018-30428-3
123. O’Mahony L, Renz H, Forbes-Blom E, Nowak-Wegrzyn A. Human milk oligosaccharides: new ways to shape the gut microbiome in cow's milk protein allergy. EMJ Allergy Immunol. 2019;4(1):48-54.

\section{Publish your work in this journal}

The Journal of Asthma and Allergy is an international, peer-reviewed open-access journal publishing original research, reports, editorials and commentaries on the following topics: Asthma; Pulmonary physiology; Asthma related clinical health; Clinical immunology and the immunological basis of disease; Pharmacological interventions and

Submit your manuscript here: https://www.dovepress.com/journal-of-asthma-and-allergy-journal new therapies. The manuscript management system is completely online and includes a very quick and fair peer-review system, which is all easy to use. Visit http://www.dovepress.com/testimonials.php to read real quotes from published authors. 